\title{
Paganisme et mysticisme dans la littérature tunisienne d'expression française: le cas d'Abdelwahab Meddeb
}

Ridha Boulaâbi

\section{(Q) OpenEdition}

Journals

Édition électronique

URL : http://journals.openedition.org/recherchestravaux/400

DOI : $10.4000 /$ recherchestravaux.400

ISSN : 1969-6434

Éditeur

UGA Éditions/Université Grenoble Alpes

Édition imprimée

Date de publication : 30 juillet 2010

Pagination : 27-40

ISBN : 978-2-84310-174-8

ISSN : 0151-1874

\section{Référence électronique}

Ridha Boulaâbi, « Paganisme et mysticisme dans la littérature tunisienne d'expression française: le cas d'Abdelwahab Meddeb », Recherches \& Travaux [En ligne], 76 | 2010, mis en ligne le 30 janvier 2012 consulté le 08 septembre 2020. URL : http://journals.openedition.org/recherchestravaux/400 ; DOI

https://doi.org/10.4000/recherchestravaux.400 
Ridha BOULAÂBI

Centre d'études du roman et du romanesque

Université de Picardie Jules-Verne

\section{Paganisme et mysticisme dans la littérature tunisienne d'expression française : le cas d'Abdelwahab Meddeb}

Cet article se propose d'étudier le récit de voyage intitulé "La tache blanche» qui ouvre Aya dans les villes, un recueil de textes dans lequel Abdelwahab Meddeb relate ses pérégrinations, réelles et imaginaires, en Jordanie, à Fès, à La Mecque. Le récit liminaire se donne à lire comme un voyage touristique en Jordanie, essentiellement à Pétra et ses alentours, que l'on peut résumer brièvement en deux parcours. Le premier parcours, touristique et collectif, réunit trois personnages, Aya, Jean et le narrateur-auteur, Meddeb, en présence d'un guide et d'un chauffeur de taxi, et il obéit à un circuit bien défini (visite des différents sites archéologiques de Pétra comme le Haut Lieu, le Tombeau de l'Urne, Kasr el Bint). Le second parcours, personnel et intériorisé, est présenté par le narrateur, dès la première page, comme une véritable quête - l'objectif étant l'identification de «la tache blanche», aperçue de loin dès l'arrivée :

Dès mon arrivée, à l'heure où le jour épouse la courbe et son déclin, je suis irrésistiblement attiré par cette haute et pourtant humble blancheur. Mon œil maghrébin assimile cette cime immaculée à quelque célébration maraboutiquet. (AV, p. 9)

Introduite par un vocabulaire religieux comme «immaculée», "célébration maraboutique», cette "tache blanche» entre ici en résonance avec un

I. A. Meddeb, Aya dans les villes, Montpellier, Fata Morgana, 1999 (désormais AV). 
autre texte, Blanches Traverses du passé dans lequel Meddeb analyse le symbole de cette couleur dans la culture arabo-musulmane à travers quelques exemples : les blanchisseuses de linge ou de laine, le badigeonnage en blanc des tombeaux et des demeures maraboutiques. Ces exemples renvoient à la recherche d'une même pureté que Meddeb associe par un jeu de mots à une pureté mystique comme on peut le lire dans l'exemple suivant :

Ainsi se décline dans l'indécision du sens l'étymologie du mot soufi, lequel vacille tantôt entre la sagesse grecque (sophia) et la référence aux Compagnons de la véranda (soffa) - ces pauvres qui hantaient la véranda sur laquelle ouvrait la maison du Prophète à Médine et qui étaient promus héros fondateurs par les premières générations soufies - tantôt entre la laine et la bure (souf) ou la tignasse (soufa) et la quête de la pureté (safa)².

En ce sens, l'objet de la quête n'est pas seulement un endroit matériel à visiter. Sa blancheur éclatante lui confere une sorte d'aura mystique qui appelle donc un voyage spirituel. Ces caractéristiques sont confirmées par la phrase qui suit :

Marque polémique des unitaires incapables de défier l'exploit païen, cette tache blanche, d'évidence apposée sur la montagne, par référence active aux Écritures, sera la cible de mon séjour, objet de quête, borne signalant l'indépassable, les confins vers lesquels mon corps avancera pour rencontrer l'arbre imaginaire qui précède le rideau d'intense lumière lequel il n'est plus possible de voir. ( $A V$, p. Io)

Cette phrase peut être lue comme une énigme et comme une clé à la fois. En effet, il s'agit d'une description truffée de références religieuses sous-jacentes : l'«arbre imaginaire» est le lotus ou le jujubier cité dans le Coran par l'expression «sidrat al mountaha» et le "rideau " n'est autre que "ma waraâ al-hijeb», cette zone indépassable qui selon les mystiques marque les limites entre le monde de Dieu et l'ici-bas. C'est dans cet endroit que tout voyage spirituel, toute communion avec le Créateur trouve sa fin. La même analyse, nous la retrouvons chez Ibn Arabî̀, dans son livre Kiteb al-asfar 'an ntaïj al-issfar (Le Dévoilement des effets du voyage $)^{4}$ :

Tous les êtres sont dans une nuée, tous étant dans la cécité et le tout est à l'image du Tout. Ce voyage dans son esprit et son sens est le passage de la transcendance au

2. Id., Banches Traverses du passé, Montpellier, Fata Morgana, 1997, p. I6.

3. Ibn Arabî est né en Murcie (560-638/II65-I240). Surnommé al-Chaykh al-akbar, il est l'un des plus grands soufis de l'islam. Il a effectué plusieurs voyages et séjours dans le monde arabo-musulman (Cordoue, Séville, Fès, Tunis, Jérusalem, Damas, La Mecque, Bagdad...). Son ouvrage le plus connu s'intitule : Tourjemène al-achwèkh (L'Interprète des désirs).

4. Ibn Arabî, Le Dévoilement des effets du voyage, texte édité, traduit de l'arabe et présenté par D. Gril, édition bilingue, Éditions de L'Éclat, 1994, Tunis, Cérès Éditions, I999 (désormais $D E)$. 
Lotus de la similitude pour que ceux auxquels s'adresse le discours divin puissent comprendre. Et ceci relève encore de cette même cécité. ( $D E$, p. I7)

C'est dans ce livre qu'Ibn Arabî a détaillé le sens et les étapes du voyage spirituel vers le royaume de Dieu, autant d'éléments qui reviendront subrepticement dans notre récit. En somme, tous ces renvois donnent le ton du voyage et l'inscrivent dans le sillage des mystiques.

Pour revenir à la notion de "clé», nous pouvons avancer l'hypothèse de travail suivante : le voyage terrestre, réel et horizontal, au milieu des vestiges antéislamiques, va de pair avec le voyage céleste, spirituel et vertical, un voyage de l'«interprète» au sens mystique du terme, qui avance au gré des révélations divines. Nous suivons donc à la fois les traces des anciens (Romains, Égyptiens et surtout Nabatéens's) et celles d'Ibn Arabî, le guide spirituel de Meddeb dans Pétra.

Il faut dire qu'Ibn Arabî et Meddeb ont tous les deux une vision ouverte et nuancée des vestiges antéislamiques, du paganisme et de l'idolâtrie, une vision qui tranche complètement avec celle de certains ultraconservateurs pour qui la véritable civilisation commence avec l'avènement de l'islam. Le voyage de Meddeb à Pétra manifeste la volonté de reconnaître l'ère païenne dans la civilisation musulmane, en se fondant sur les faits archéologiques, sur les analyses philologiques et sur les interprétations théologiques d'Ibn Arabî. Toutefois, une différence de taille distingue les deux hommes. En effet, le maître, Ibn Arabî, est un homme de religion, un mystique qui a dédié sa vie et ses œuvres à l'adoration de Dieu. Quant au disciple, Meddeb, il se présente comme un laïque incroyant. Il s'intéresse non pas à la religion en tant que telle, mais à la beauté des récits qu'elle a pu engendrer. Au delà de toutes les références directes à Ibn Arabî, le texte de Meddeb dialogue en profondeur avec le maitre akbarien notamment à propos du voyage, qu'il s'agisse de sa forme, de ses étapes ou de ses résultats. Il serait donc intéressant de voir de près comment le voyage touristique d'un laïque suit le cheminement du voyage spirituel d'un mystique, pour aboutir au même résultat : la reconnaissance des civilisations païennes.

5. Le pays d'origine des tribus nomades des Nabatu reste difficile à déterminer. Une étude récente a cherché leur origine entre le Koweït et la Mésopotamie. Les études ont attesté également leur origine arabe à travers l'onomastique de leurs divinités et surtout l'utilisation de l'article al. Ces tribus adoptèrent, après leur expansion au nord-est de l'Arabie, l'araméen comme langua franca. Au v ${ }^{\mathrm{e}}$ siècle av. J.-C., elles s'emparèrent de Pétra qui devient la capitale du royaume. Malgré les nombreuses guerres qui secouèrent le Proche-Orient, elles réussirent à préserver leur indépendance face aux grandes puissances conquérantes : les Ptolémées, les Séleucides, les Asmonéens et les Romains. Ce n'est qu'en Io6 apr. J.-C. que l'empereur Trajan ordonna l'annexion du royaume nabatéen à l'empire pour former la province d'Arabie. 


\section{Vers un voyage mystique}

Le récit dans sa globalité semble se fonder sur le sens arabe du mot safar («voyage»), tel qu’il est expliqué par Ibn Arabî :

Le voyage a été appelé safar parce qu'il dévoile (yusfiru) le caractère des hommes, faisant apparaître les caractères blâmables et louables que tout homme recèle en lui. On dit aussi : la femme a dévoilé son visage (safarat 'an wajhihâ) quand elle enlève son voile et qu’apparaît sa beauté ou sa laideur. ( $D E$, p. 2I)

L'association entre les deux sens de «cheminement» et de «révélation" ponctue tout le texte de Meddeb. La promenade du voyageur-interprète parmi les vestiges nabatéens s'avère systématiquement une occasion de découvrir un sens caché. Néanmoins, pour que ces réalités invisibles deviennent visibles, le voyageur doit se soumettre à un rituel qui s'apparente à celui des soufis. Il doit ainsi préparer son corps à la réception de ces révélations. En effet, Meddeb évoque dès les premières pages la danse des derviches pour mettre son corps en correspondance avec le lieu à visiter :

Ainsi vont les pensées qui me hantent avant de lancer mon corps dans une approche légère, dansante, sur les pieds, dans la quiète ferveur de l'heure vespérale qui me voit avancer le long du wadi rocailleux ${ }^{6}$. ( $A V$, p. Io)

La même association entre le corps, le lieu et la révélation revient dans beaucoup de textes. Meddeb l'hérite en quelque sorte d'Ibn Arabî qui emploie les mêmes termes ("correspondances", «état", «instant", "écoute») comme on peut le lire dans l'exemple suivant :

Sache que l'être humain possède en lui des correspondances (munâsib) avec chaque élément de l'univers et que chacune en elle est en relation avec celles de l'homme dans ses aspects les plus apparents. L'état (hâl), l'instant (waqt) et l'écoute $(\operatorname{sam} \hat{)})$ lui sont attribués en raison de correspondances que n'ont pas les autres êtres créés, puisqu'il possède des affinités multiples avec ces correspondances, à cause des aspects nombreux exigés par son essence?.

6. Cette correspondance essentielle entre le corps et l'espace est à lire également dans un autre livre de Meddeb, L'Exil occidental : "Je dis ce que le corps enregistre. C'est ma façon d'être présent au monde, dans ce que le lieu révèle. Ou dans ce qui se révèle entre la conjonction d'un corps et d'un lieu. Ainsi, se concrétise l'errance à travers l'actualisation soumise aux normes qui nous disséminent pour nous rendre disponibles à la discontinuité de notre espace-temps. [...] Les images et les figures dissolues conduisent à la jouissance des traces et des vestiges, comme pour ne point oublier que le monde ressemble à une maison abandonnée, dont l'approche alourdit les épaules et serre la poitrine d'un corps qui reste marqué par le rapt et le frisson que procurent les heures intermédiaires où il se consacre au vin, aux mets, à la fleur, au fauve, au rapace [...]» (L'Exil occidental, Albin Michel, 2005, p. I3.)

7. Ibn Arabî, Tourjemène al-achwèkh (L'Interprète des désirs), présentation et traduction de M. Gloton, Albin Michel, coll. «Spiritualités vivantes», 1996, p. I89. 
Une fois le rituel respecté, le sujet voyageur est prêt à arpenter le site et à recueillir toutes formes de révélation grâce à cette osmose entre l'esprit, le corps et le lieu.

\section{Le voyage proprement dit : les étapes}

Pour comprendre la logique de ce parcours qui emprunte ses traits à celui d'Ibn Arabî, il nous faut d'abord dire quelques mots sur le maître à penser afin d'analyser ce glissement permanent du mystique au laïque, du sacré au profane. En effet, pour résumer, selon Ibn Arabî, il existe trois sortes de voyages. Le premier est le Voyage à partir de Dieu ou as-safar 'an al-illeh. Il concerne tous les êtres humains, car il s'agit de notre première descente $\mathrm{du}$ paradis et du royaume divin vers le monde terrestre, loin de Dieu. Le deuxième, nommé le Voyage vers Dieu ou as-safar ila al-illeh, adopte le mouvement inverse, c'est-à-dire une remontée vers Dieu par laquelle, et grâce à l'assistance d'un guide, l'homme accomplit le voyage spirituel. Le troisième et dernier voyage, as-asfar fi-l-illah, est un voyage en Dieu, défini comme une réalité difficile à atteindre, intériorisée et sans fin. Le voyageur vit avec ses semblables, mais à l'intérieur de soi, et il communie en permanence avec Dieu. Si l'on applique ce schéma aux pérégrinations de Meddeb tout en remplaçant Dieu par l'islam (il s'agit d'une hypothèse de lecture bien entendu), on obtient trois types de voyage qui s'entrecroisent tout au long du récit.

\section{Voyage à partir de l'islam (as-safar 'an al-islam)}

Dans cette première étape, Meddeb opte pour le même mouvement de descente. Il descend de l'islam et du dieu unique vers ce qui l'a précédé, c'està-dire vers la période antéislamique polythéiste. On remarque que le narrateur recourt, pour justifier ce rapprochement, à une "curieuse métaphore" qui met justement en évidence cette exploration des profondeurs :

[...] des premières tombes, petites tours isolées, taillées à même le roc, ou façades monumentales sculptées dans le relief, capturant la lumière du crépuscule et creusant une ombre épaisse dans l'étagement où succèdent aux obélisques les pilastres et les frontons, saillies rongées par le vent et le sable, comme érodées par les courants diurnes d'une mer qui s'était retirée. Curieuse métaphore qui s'impose dans mon esprit et qui associe cet espace aérien et de montagne à un fond de mer dont l'eau se serait évaporée, sel picotant mes yeux jusqu'aux larmes qui se mettent en ruissellement précoce et instantané du corps, se laissant emporter par l'hallucination qui transfigure en fossiles les vestiges de l'œuvre humaine. $(A V$, p. II) 
En attirant notre attention par des mots comme "curieuse métaphore qui s'impose", Meddeb nous invite discrètement à lire son message au second degré, d'une manière presque allégorique (une invitation que son maître Ibn Arabî a toujours pratiquée pour éviter d'être taxé d'hérésie par ses ennemis). L'espace aérien serait donc le Ciel divin de l'Islam et le fond de mer serait le bain culturel dans lequel il est né. Des termes comme "fossiles» et "vestiges» prennent une acception particulière ici puisqu'il s'agit justement de visiter les empreintes laissées par les civilisations antérieures. Cette métaphore de la descente vers les profondeurs historiques est renforcée dans le récit par un champ lexical très connoté comme "ténèbres", "obscurité", "fond", "tunnel». Le mot arabe nommant cette période païenne, al-jahiliya, littéralement "l'ignorance», ne renvoie-t-il pas à ces mêmes ténèbres ? Dans le Coran, le mot est étroitement associé à dhouloumett, c'est-à-dire "obscurité", par opposition à l'islam comme nour, c'est-à-dire «lumière».

\section{Voyage vers l'islam (as-safar ila-l-islam)}

Au cours de cette deuxième étape, le voyageur-interprète se fixe comme point de départ les sites nabatéens de Pétra et, comme point d'arrivée l'islam, luimême. Il sera guidé dans cette remontée dans le temps par l'ombre d'Ibn Arabî qui plane sur ce parcours. Comme dans le voyage spirituel du soufi, le voyage de Meddeb connaîtra lui aussi des étapes, des paliers ou des «stations» (mawaquifs), pour emprunter le terme aux mystiques. En ce sens, les vestiges rencontrés lors de ce parcours vaudraient comme autant de stations conduisant à un sens caché. Ce mouvement de remontée s'exprime par un vocabulaire explicite comme "élévation ", "montons ", "escaladons", "étroite montée», "ascension" (répété plusieurs fois). Dans certains passages, le mot "ascension» est associé au terme $m i$ 'râj, c'est-à-dire l'ascension du prophète Mohamed dans le royaume de Dieu.

\section{Voyage dans l'islam (as-safar fi-l-islam)}

Dans notre récit, ce troisième type de voyage s'effectue à l'intérieur même de la pensée du sujet. En effet, comme nous le verrons, Meddeb tentera de lire la survivance des civilisations païennes à travers son propre prénom et à partir des effusions de son corps au contact de l'espace païen. Le sujet-voyageur, en

8. "Miraj, litt. “ascension”. Légende hagiographique, mais rapportée par le Coran, selon laquelle le Prophète aurait effectué, sur un cheval fabuleux appelé al-bouraq ou bourak, une ascension (miraj) au Septième Ciel en l'an 6I5, le 27 du mois de rajab. Il serait parti de la Mecque, où se trouve la Mosquée sacrée, fit une étape à Jérusalem, lieu de la Mosquée extrême (peut-être le Paradis) et rejoignit le Ciel après.» (M. Chebel, Dictionnaire des symboles musulmans, Albin Michel, coll. «Spiritualités vivantes», I995, p. 27I.) 
relation permanente avec l'époque païenne, serait donc une preuve vivante et irréfutable de cette continuité entre les deux aires culturelles.

\section{Le Voyage vers l'islam : des sites archéologiques ou des stations mystiques?}

La promenade est une rencontre directe avec les ruines. Traces du passé, elles ont un message à délivrer. Toute rencontre devient donc une occasion de révélation, une forme de réminiscence du passé dans le présent, de l'ancien dans le moderne, et toute révélation est vécue comme une épiphanie, un sentiment que Meddeb? traduit par le mot soufi de tajalliat :

J'utilise le mot «épiphanie» dans le sens que lui donne le romancier irlandais James Joyce, sens qui suscite en moi l'écho de la notion soufie de tajalli, laquelle décrit l'opération révélant l'invisible dans les choses concrètes; il s'agit des visions et des révélations qui transfigurent les heures urbaines du promeneur et qui instaurent la poésie de la ville (MI, p. 139).

La rencontre avec les traces païennes déclenche chez Meddeb ce qu'il appelle, dès les premières pages, "la trame de la fiction" ( $A V$, p. Io). Toute rencontre donne naissance à une association métaphorique entre des éléments archéologiques appartenant à différentes civilisations et des récits du Coran, minutieusement choisis. Les exemples sont souvent construits selon le schéma suivant : attirance pour une trace païenne, révélation sous la forme d'une épiphanie ou tajalliat, récit de cette épiphanie à partir de versets coraniques, pour aboutir à une conclusion rappelant la démarche soufie. À preuve l'exemple qui suit :

Dans le silence et la solitude qui favorisent le soliloque, mon attention se raffermit à la vue des bétyles qui me transportent dans l'atmosphère de la croyance contre laquelle avait protesté l'islam. Le culte des pierres dénoncées par le Coran propose son décor sur les parois qui assaillent mon regard. "Avez-vous vu al-Lât, al-uzzâ / Et l'autre, Manât, la troisième ?» Qui reconnaîtrais-je en ce bloc taillé à l'intérieur d'une porte, elle-même sculptée dans un cube partiellement détaché de la falaise ? Reconnaîtrais-je une des déesses honnies en ce volume géométrique qui déborde à peine l'aniconisme en suggérant les traits d'un visage à travers deux carrés séparés d'une barre? ( $A V$, p. I8)

D'emblée, la correspondance entre l'état du voyageur («solitude») et du lieu ("silence») favorise la rencontre visuelle avec les bétyles et déclenche un retour au panthéon arabe antéislamique par l'invocation de ses célèbres

9. A. Meddeb, La Maladie de l'islam, Seuil, coll. «La couleur des idées», 2002, (désormais $M I)$. 
déesses vénérées également par les Nabatéens de Pétra al-Lât ${ }^{10}$, al-uzzâa ${ }^{\mathrm{II}}{ }$ et "Manât $t^{12}$ ». Par le mot "bétyles», en arabe nusub, Meddeb désigne les pierres dressées sur lesquelles on versait le sang des victimes immolées aux idoles ${ }^{13}$. Le voyageur est sensible au minimalisme du trait et à l'aniconisme des sculptures chers aux Nabatéens, qui, en Sémites conservateurs, ne représentaient pas leurs dieux sous forme humaine mais, seulement sous la forme d'un bloc, quadrangulaire, appelé «bétyle» ou "habitacle de Dieu »" ${ }^{\mathrm{T}}$. Mais, le lecteur sait-il que cette phrase évoquant les idoles païennes correspond dans le Coran au verset i9 de la sourate "l'Étoile ${ }^{15}$ " ? En effet, sorti de son contexte (une mise en garde contre l'idolâtrie), le verset est mis au service d'une reconnaissance de ces bétyles dans le monde contemporain. L'invocation successive de références païennes se conclut par un vocabulaire mystique "perçois", "trouble apparition", "se clarifie» et par une citation directe de Niffari ${ }^{16}$, un soufi irakien souvent cité par Ibn Arabî.

Après le passage par plusieurs stations (la Tombe de l'Urne et le dialogue entre Salomon et la huppe ${ }^{17}$, le Haut Lieu et le sacrifice d'Abraham ${ }^{18}$ ),

IO. Al-Lât : nom des trois divinités les plus vénérées du panthéon arabe antéislamique. La signification reste imprécise. Elle est parfois considérée comme parèdre d'Allah ou de son patronyme IL/EL. Al-Lât serait une forme féminine d'Allah ou al-Ilâh, dieu anonyme du panthéon arabe préislamique.

II. Al-uzzâ : «la Toute-Puissante», déesse de la confédération kurayshite.

I2. Manât : une des plus connues et des plus anciennes des divinités du panthéon arabe antéislamique. Elle est incarnée par un rocher sacré, puis par une statue (idole). Son nom est lié à la mort et au destin (d'où le mot arabe mannia qui signifie «mort».)

13. Il arrive parfois chez les nomades que le nusub soit pris comme symbole de la divinité. Nusub devient peu à peu l'équivalent de sanâm, c'est-à-dire «idole». Le culte des pierres, profondément ancré chez les Arabes du Hijâz, ne s'est pas transformé aussi rapidement qu'ailleurs en culte de statues. C'est au milieu du $\mathrm{III}^{\mathrm{e}}$ siècle de notre ère que des influences nabatéennes et syro-palestienniennes se sont exercées pour promouvoir, dans les cultes urbains, l'évolution plastique du panthéon arabe. Pour plus d'information, voir Dictionnaire encyclopédique de l'Islam, Maisonneuve et Larose, 1982, t. VIII, p. I57.

I4. "Pétra et le royaume des Nabatéens", Les Dossiers d'archéologie, n I63, septembre I99I, p. 22.

15. Le Coran, essai de traduction, par J. Berque, Albin Michel, coll. "Spiritualités vivantes», 1995, p. 575 .

16. Muhammad Ibn Abd-al-Jabbar al-Hasan al-Niffari serait mort en l'an 354 de l'Hégire (965 apr. J.-C.), soit une quarantaine d'années après Husayn Ibn Mansur Hallaj, le martyr du soufisme. Son surnom lui serait venu du nom du village de Al-Niffar, dans la région de Kufa en Irak. Il a laissé deux ouvrages : Al-Mawaqif(Les Stations) et Al Mukhatabat (Les Adresses).

I7. Dans les pages 2I et 22, Meddeb relate la montée vers la «tombe de l'Urne» qui est aussi le lieu d'une divagation, d'une reconstruction fictive d'un dialogue entre Salomon et la huppe au cours duquel on évoque la reine de Saba. Ce dialogue est inspiré pour ne pas dire calqué de la sourate «Les fourmis» (versets is à 44). Meddeb ne mentionne pas la sourate clairement, mais se contente d'expressions comme «bribes coraniques». Le récit finit également par la phrase : «il me révèle» $(A V$, p. 22) qui rappelle la méthode soufie.

I8. Dans les pages 24 à 26 , le voyageur atteint le plateau du «Haut Lieu», sur la montagne du sacrifice». Les correspondances entre le lieu, le corps et les révélations sont ingénieusement 
le voyage finit par l'identification de la tache blanche tant recherchée. Il s'agit du mausolée d'Aaron à Pétra, le lieu où reposerait le corps du frère de Moïse, enseveli sur cette montagne lors de l'Exode des Hébreux au XIII ${ }^{\mathrm{e}}$ siècle av. J.-C. C'est dans cette "mosquée homonyme» ( $A V$, p. 27) que Meddeb reprend l'histoire des deux prophètes (p. 35) en jouant sur la permutation des noms arabes "Moussa" et "Haroun" et de leurs équivalents bibliques «Moïse» et "Aaron». Il enchaîne directement avec l'épisode du veau d'or, évoqué par le Coran, et repris dans le voyage d'Ibn Arabî. La "tache blanche» correspond donc à une forme de syncrétisme religieux très cher à Meddeb. L'imbrication des trois religions monothéistes se lit à travers un jeu sur les noms tantôt arabes, tantôt bibliques de Moïse et d'Aaron, ou à travers l'association de "mosquée» et de "crypte». À tout cela s'ajoute l'épisode du veau d'or qui renvoie à l'idolâtrie, donc à l'époque païenne.

Que retient-on, in fine, de toutes ces révélations?

D'abord, toutes ces stations visitées par le touriste-interprète embrassent les différentes civilisations qui cohabitent dans cet espace. Les influences architecturales et théologiques dont témoignent encore les vestiges de Pétra montrent fort bien la réussite du syncrétisme nabatéen qui a su réunir les Grecs, les Romains, les Arabes, les Hébreux et les Égyptiens dans une seule et même civilisation. C'est donc dans ce lieu syncrétiste que l'islam a germé, un lieu que certaines âmes bien pensantes continuent à occulter selon Meddeb ${ }^{19}$. Il ne s'agit pas pour lui de s'opposer à l'islam, mais de rétablir une vérité historique, archéologique et de reconstruire les ponts entre l'islam et les civilisations qui l'ont précédé. Ensuite, l'association des récits coraniques et des vestiges visités souligne cette continuité, sur le plan littéraire. Sur le plan théologique, les interprétations d'Ibn Arabî reprises ici par Meddeb offrent une lecture novatrice, analysant le culte nabatéen comme une forme inférieure du monothéisme. Les païens, selon Ibn Arabî, multiplient les idoles, certes, mais ils attribuent le pouvoir suprême à une seule et unique divinité. Force est de reconnaître que ces analyses nuancées s’appliquent parfaitement

développées à ce stade de la promenade. Le lieu du «Sacrifice» qui renvoie forcément à Abraham acquiert d'un coup une dimension particulière, la montée devient de plus en plus solennelle, oppressante. La réaction du corps est à la hauteur des symboles que recèle le lieu visité : «vertige», "corps vacille», "genoux flageolent», "jambes tremblent", "révélation du sentiment coupable», "expiais-je», "une force qui ne m'appartient plus». Le corps revit en quelque sorte l'épisode du sacrifice, remémore les moments de doute d'Abraham. Le souvenir redonne au lieu sa dimension sacrée que le corps détecte. Le récit finit comme la légende par l'attente d'un miracle : «On voudrait qu'un miracle lui épargnât la précoce usure qui guette l'humain soumis au régime des pierres.» ( $A V$, p. 25$)$

19. "Dans cette prétention, ils ont été précédés par leurs ancêtres nabatéens et païens qui avaient rassemblé la Grèce et l'Égypte dans le décor de leur façade $[\ldots]$ » ( $A V$, p. I9) 
à la civilisation nabatéenne. Car les découvertes archéologiques ont montré que les croyances des caravaniers nabatéens avaient en quelque sorte préparé la voie aux grandes religions monothéistes : par leur dévotion à un dieu Ehayw, ils partageaient avec les juifs la croyance en Yahweh. En professant la naissance de leur dieu Dushra $\hat{a}^{20}$, fils de la mère vierge Al-Uzza-Aphrodite, ils ont facilité l'expansion du christianisme qui a ajouté au mystère de la virginité celui de la rédemption. En ce qui concerne l'islam, sans croire au monothéisme, les Nabatéens adoraient Allah al-Ilah, que le Coran a débarrassé des divinités secondaires.

\section{Le voyage dans l'islam}

Ainsi arrive-t-on jusqu'à l'islam. Après avoir creusé le passé, Meddeb continue son voyage païen au cœur de lui-même, c'est-à-dire dans l'intimité du sujet voyageur. Au cours du voyage intériorisé, nous lisons la description suivante :

L'étroitesse oppresse mon corps. C'est comme si j'étais entre les deux pierres de la meule. J'entends mon cœur battre. Mon souffle cogne sur les parois hautes et droites. Mon haleine imprègne de ses effluves les niches et les stèles qui célèbrent les morts. Est-ce le hasard si ma respiration entre en résonance avec les nefesh, mot sémitique qui désigne les stèles en nabatéen et qui signifie en arabe, le souffle, l'individu, le moi, le principe vital, le sang, l'essence de la chose, le mauvais œeil, la grandeur, le haut rang, la dignité ? Cette polysémie profuse n’honore-t-elle pas la vocation et le principe de la stèle ? N'esquisse-t-elle pas un réseau cohérent pour le discours? Le souffle cesse d'émaner de l'individu, le sang s'y coagule, le principe vital s'en retire, alors la lettre glorifie la personne dont l'âme déserte le corps pour maintenir son nom dans la grandeur et la dignité qui jaillissent sur ceux qui en jouissent encore et les protégeraient des coups du sort. ( $A V$, p. I3-I4)

Encore une fois, nous retrouvons les mêmes correspondances entre l'état, le lieu et les révélations. Meddeb part de son corps et de sa réaction dans le lieu visité (respiration, haleine, oppression) pour arriver à la trace : les stèles nabatéennes célébrant les morts. Le développement philologique révèle la filiation entre nefesh et nefesh. Le choix s'avère extrêmement judicieux compte tenu des significations religieuses et mystiques que recèle un tel mot. Meddeb se

20. Dhûshara : surnom arabe d'un dieu emprunté aux Nabatéens et dont l'appellation araméenne est $d s h$ (dich'r). C'était le dieu local du massif Shara, l'extrémité méridionale de la chaîne de montagnes au sud-est de la mer Morte. On lui offrait des sacrifices sanglants. Identifié à Dionysos comme l'atteste Hérodote, il est représenté sur de nombreux tombeaux nabatéens par un aigle aux ailes déployées. Le bétyle de Dhûshara s'appelle kaâba et se trouvait selon l'historien Ibn Ichem dans un hima, enceinte sacrée appelée aussi haram. 
lance dans une rêverie sur le mot nefesh et ses différentes acceptions. Le souffle nefesh cesse, l'âme an-neffs déserte le corps, et seule reste la lettre inscrite sur la stèle nabatéenne signifiant nefesh. Toutefois, comme après toute mort, la vie renaît. Meddeb, en poète, insuffle la vie à la stèle en entrant en contact avec elle par son souffle, et c'est à travers son corps que la stèle nabatéenne retrouve son âme, et que Meddeb retrouve métaphoriquement les liens entre islam et traces païennes. Il applique la même méthode aux deux composantes de son propre prénom :

Toute manifestation bilingue m'émeut, j'y cueille une preuve supplémentaire qui légitime mon état; chaque fois que j'ajoute un chaînon à ma généalogie de sujet bilingue, je me sens enrichi; ainsi j'additionne les siècles et les millénaires pour les intégrer à mon âge. «Maqbara choisie par Abdmank fils d'Akayus. » La résonance arabe des mots accélère mon identification. Maqbara, qui signifie ici sépulture, veut dire en arabe "cimetière». Et dans $A b d$, serviteur, je reconnais le schème sur lequel est construit mon propre nom. La formation de mon anthroponyme, obéissant au théocentrisme islamique, à la soumission de la personne au nom divin, ce nom théophore, je l'hérite de l'ère païenne. ( $A V$, p. I5-16)

$A b d$, est la racine de presque tous les noms propres dérivés des noms sacrés de Dieu; Abdelwahab signifie littéralement le «serviteur du Donateur». La particule dénotant la soumission à Dieu offre des parentés avec l'ère païenne et témoigne, selon l'auteur, de la continuité entre les deux civilisations. De la même manière, il retrouve dans le mot nabatéen wahballâhi la seconde moitié de son prénom Waheb, dérivé du verbe arabe wahaba qui signifie «offrir» :

J'emprunte à la même source la traduction : "Ceci est le massabâ / stèle de Bosra qu'a fait Wahballâhi.» Cette citation serait pédante ou vaine si elle ne révélait pas une relation entre le nom de l'orant nabatéen et mon propre nom arabe. Je recueille cette ressemblance dans l'ignorance des langues. Malgré ma méconnaissance de l'araméen, je reçois Wahballahhi dans une résonance arabe qui veut dire "don de Dieu», et dont le premier segment (Wabba) me fut donné en petit nom quand j'étais enfant : c'est un diminutif forgé à partir de mon prénom qui signifie le serf, le serviteur, le suppôt, peut-être le sujet du Donateur, un des noms de Dieu. Les deux noms sont théophores, ils ont le don divin en partage. Après l'analogie morphologique du nom reconnue dans l'inscription bilingue, après la présente identité de sens, s'établit la généalogie nabatéenne d'un nom arabe toujours porté par des vivants. ( $A V$, p. 29)

Le prénom de Meddeb, Abdelwahab, s'avère donc la trace vivante de la langue et de la cosmogonie nabatéennes : l'ère païenne continue à vivre dans et par la langue arabe, la langue du Dieu unique, Meddeb devient un maillon vivant et actif de cette longue chaîne qui relie l'islam à ses ancêtres païens. Le narrateur se transforme lui-même en un vestige païen, en une trace qui vient de loin et qui témoigne de cette continuité. Plus important encore, il est le garant 
même de cette survivance à travers son nom, partagé entre les deux traditions. Les traces du paganisme ne résident plus en dehors du sujet parlant : elles sont en lui, à la fois dans son corps et dans les lettres qui glorifient son nom. Si le but d'un voyage soufi comme celui d'Ibn Arabî est d'atteindre, par le corps et par l'esprit, cette communion intime avec le Dieu unique, le but du voyage profane de Meddeb est d'atteindre, également par son corps et par son esprit, cette communion intime et intériorisée avec les dieux antéislamiques qui célèbrent l'Un. Le délire des mots qui rappelle celui des mystiques atteint son paroxysme par la fusion des voyages spirituel et païen à la fin du récit :

Ce qui est reproché aux païens n'est pas la destitution de l'Un, mais la concurrence qu'Il subit en Lui associant d'autres figures : parèdres, intercesseurs, protecteurs, tuteurs, idoles. Les païens s'étaient arrêtés à la pluralité des formes dans leur rapport à la divinité. Le prophète les convia à adorer un Dieu unique, à connaître sans qu'Il soit vu [...] Dans la crainte et le frisson du soir approfondissant la ténèbre du sanctuaire, je m’entends psalmodier : «Paix sur al-Lât, sur Manât, paix sur al-Uzzâ, sur Dhûshara, paix sur le Dieu invisible qu'ils rendent visible.» $(A V$, p. 39)

L'invocation de l'Un, de Lui, du Il, met Allah en relation directe avec le panthéon antéislamique et nabatéen, faisant ainsi du sujet délirant un sujet syncrétiste qui reçoit, tel un mystique mais païen, des révélations touchant à des civilisations diverses et variées.

\section{Les leçons du voyage}

Les leçons à tirer de ce récit sont surtout implicites. Elles s'inscrivent dans un projet plus général, plus politique, qui vise à combattre l'obscurantisme religieux grâce à des figures de l'islam des Lumières comme Ibn Arabî. Presque tous les sujets abordés ici, sur le mode de la narration et dans le cadre d'un récit de voyage, trouvent des échos dans les essais d'un Meddeb clairement et directement engagé à la fois contre une vision violente de l'islam et contre sa diabolisation par un certain Occident conservateur. En effet, la réhabilitation de l'ère païenne constitue une réponse à ceux qui nient l'histoire et vont jusqu’à détruire toute trace archéologique antérieure. Dans La Maladie de l'islam, Meddeb dénonce la destruction des bouddhas de Bamiyan par les talibans et rappelle à travers l'esprit de tolérance que prêche Ibn Arabî que l'islam comme croyance et comme civilisation est étranger à ce genre de violence :

Les bouddhas géants, sculptés à même les parois de la montagne entre le troisième et le quatrième siècle de notre ère, restaient signifiants pour une pratique religieuse encore vive. Sur le bord où je me situe, toutes les croyances méritent d'être considérées : c'est un enseignement que j'extrais du soufisme, notamment de la tradition 
akbarienne, élaborée dans le cadre de la foi islamique d'Ibn Arabî (II65-I240), le maitre andalou qui recommande d'être "de hylee pour qu'en vous prennent forme les croyances toutes». C'est-à-dire que pour le soufi natif de Murcie, le sujet islamique a la capacité d'intérioriser toutes les formes de croyances et de cheminer avec leur vérité sans chercher à les réduire ou à les escamoter ( $M I, \mathrm{p}$. I49).

Il n'hésite pas non plus à dénoncer les agissements de certains pays de la péninsule arabique contre les sites archéologiques accusés de corrompre la croyance en Dieu :

Mon ami, le poète Salah Stétié, m'a affirmé qu'il a appris en visitant l'Arabie que, dès qu'apparaît le moindre indice archéologique se rapportant à l'histoire de l'islam premier ou à des époques postérieures, il est immédiatement couvert d'une chape de ciment. Pour préserver sa croyance, le wahhabite n’hésite pas à porter atteinte aux vestiges qui font la civilisation, dans le seul but de ne laisser aucune chance à la redoutable confrontation du mythe avec le document historique (MI, p. 69).

En outre, l'évocation de l'épisode du sacrifice d'Abraham se donne à lire comme la réponse du Coran à ceux qui appellent à la mort. Meddeb ne fait plus appel au récit, mais cite directement les versets et commente la valeur symbolique de la parabole du sacrifice. La conclusion est sans appel :

L'Islam ne connaît pas le sacrifice humain. [...] Le sujet d'Islam célèbre chaque année le sacrifice d'Abraham, fondé sur une opération de substitution où Dieu rachète le fils par la bête. (MI, p. I82)

De la même manière, le rappel des influences artistiques, notamment architecturales, s'adresse surtout à ceux qui voient dans toute imitation scripturale ou artistique une forme d'idolâtrie. Pour cela, Meddeb oppose le conservatisme d'Ibn Taymiyâa à l'ouverture d'Ibn Arabî (Ibn Taymiyâ a attaqué plusieurs fois Ibn Arabî en le traitant d'hérétique à propos de ces sujets) :

Le même Ibn Arabî tempère la réfutation monothéiste de l'idolâtrie; il la déniaise même. Le culte des images n'est pas considéré comme négligeable, il peut apparaitre à l'intérieur de la croyance comme un degré inférieur de l'adoration; l'orant est soumis à une hiérarchie dans ses exercices spirituels : l'adoration qui sollicite l'image reste inférieure, mais elle n'est point nulle. Ce qui caractérise les croyances, ce qui les unifie et les authentifie au-delà de leur différence formelle, c'est qu'elles sont toutes fondées sur la passion $\left(a l-h^{\prime} a w a ̂ a\right)$. Et quel que soit l'objet de

2I. Dans La Maladie de l'islam, Meddeb nous fournit l'explication suivante : « [...] c'est le même mot grec qu'utilise Ibn 'Arabi en arabe (hayûli) pour désigner la matière qui accueillera la forme» (p. I49).

22. Ibn Taymiyâ, de son nom complet Taqī ad-Dīn Abu 'l Abbās Ahmad ibn 'Abd al-Halīm ibn 'Abd as-Salām ibn 'Abdullah ibn Taymiyya al-Harrānī (I263- I328), est un, apparenté au madhhab «doctrine» hanbalite. Il est souvent considéré comme l'un des plus grands théologiens de tous les temps et l'un des maîtres du traditionalisme. Selon Meddeb, il est le maître-penseur de la mouvance wahhabite. 
l'adoration (pierre, arbre, animal, représentation humaine, astre, ange), l'orant reste toujours confronté à une forme imagée de la divinité (MI, p. I5I).

Enfin, l'inscription du corps au centre même de tout voyage, qu'il soit mystique ou laïque, est une manière de rappeler qu'il existe un islam de jouissance et de vie, qui fait du culte du corps une pratique religieuse, mystique et poétique.

Mais, pourquoi voyager ? Pourquoi aller aussi loin, jusqu'aux Nabatéens païens du v viècle av. J.-C., quand tout est contenu dans le mot arabe Aya qui renvoie à la fois au titre (Aya dans les villes) et au personnage féminin qui accompagne le narrateur? Ce terme polysémique condense pour ainsi dire tous les sujets que nous venons d'aborder. En effet, aya signifie d'abord «trace visible et concrète élevée à dessein pour signaler quelque chose, comme des pierres tombales». Aya est ensuite signe visible de l'invisible puisque le mot «verset coranique». Les traces écrites du Livre saint disent Allah l'unique et l'invisible. Aya renvoie aussi à la beauté parfaite, ayatoun fil jamali, s'appliquant à cette femme qui suit Meddeb et le guide dans ses réminiscences. Aya représente enfin le personnage-trace, incarnation non seulement de la beauté physique, mais aussi d'une beauté poético-mystique qui réunit à la fois la lettre et l'esprit (le culte de cette femme énigmatique chez Meddeb rappelle le culte d'Al-uzza, littéralement «la Toute-Puissante»). Chez Abdelwahab Meddeb, tout est une histoire de résonance et tout résonne dans le nom Aya. 